\title{
Über die im weiblichen Geschlechte ungeflügelte und schwingerlose Dipterengattung Epidapus Hal.
}

\author{
Von Karl Czižek, Brünn. \\ (Mit 8 Textfiguren.)
}

Die Gattung Epidapus wurde von Halida y. ${ }^{1}$ ) für das Weibchen einer vollkommen flügel- und schwingerlosen Fliege aufgestellt, welche er 1837 in Curtis Guide aus Irland unter dem Namen Chionea venatica beschrieben hatte.

Schon 1778 hat aber Degeer²) ebenfalls die Beschreibung des Weibchens eines flügellosen Dipterons, das er Tipula atomaria nannte, veröffentlicht.

Sowohl Tipula atomaria Deg. als auch Epidapus venaticus Hal. werden dann später in der Literatur nur selten erwähnt und fast alle Notizen beschränken sich darauf, die Stellung der beiden Arten im Dipterensystem festzulegen.

I acquart ${ }^{3}$ ) meint, daß Tipula atomaria Deg. infolge der fehlenden Flügel zwar eine Gattung in der Nähe des Genus Chionea zu bilden scheine, aber durch die Anzahl der Fühlerglieder und die knotige Form derselben sich den Cecidom yi d en bedeutend nähere.

Bigot ${ }^{4}$ ) erkennt die Degeersche Art als selbständige Gattung, gibt ihr den Namen Atomaria Degeerii, reiht sie aber zu den Chi on een.

Schiner ${ }^{5}$ ) stellt die Gattung Epidapus Hal. zu den Mycetophiliden, unmittelbar vor die Gattung Sciara, also zu der heutigen Familie der Lycoriidae (Sciaridae). Er vermutet, daß das bekannte Epidapus-Weibchen zu einer geflügelten Sciara-Art gehöre, und bemerkt, daf Winnertz, Haliday und Westwood derselben Ansicht seien.

Die gleiche Stellung weist auch Winnertz ${ }^{6}$ ) der Gattung Epidapus Hal. zu. Err stellt sie zu den Sciarinen, zwischen die Gattungen Bradysia Winn. und Zygoneura Mgn.

1) Walker, Ins. Brit. Dipt., I., 7 (1851).

$\left.{ }^{2}\right)$ Mémoires pour serv. à l'histoire des Insectes. Tome VII, p. 602 (1778). Aus dem Französischen übersetzt und herausgegeben von Goeze, Nürnberg 1783. Den Auszug aus dieser Übersetzung (der Originaltext war mir nicht zugänglich) und die Abschrift der bezüglichen Stellen aus Macquart verdanke ich der Freundlichkeit des Herrn A. Kuntze, dem ich für seine Liebenswürdigkeit auch an dieser Stelle bestens danke.

$\left.{ }^{3}\right)$ Histoire naturelle des Insectes. Diptères. I. S. 119 (1834).

$\left.{ }^{4}\right)$ Annales Soc. Entomol. de Fr., Serie III, T. II. (1854), S. 454.

$\left.{ }^{5}\right)$ Fauna austriaca. Die Fliegen. II (1864), S. 416.

$\left.{ }^{6}\right)$ Beiträge zu einer Monographie der Sciarinen. Wien (1867), S. 182.

Wien. Entom. Zeitung, XXXIV. Jhg., Heft VIII-X, Festschr. f. Reitter (22. Okt. 1915). 
Mik ${ }^{1}$ ) spricht Tipula atomaria Deg. als einen Epidapus an und stellt diese Gattung gleichfalls zu den Sciariden. Auf Grund der Beschreibungen Halidays und Degeers untersucht er die Artrechte von venaticus Hal. und atomarius Deg., wobei er zu dem Schlusse kommt, daß beide vorläufig als eigene Arten $\mathrm{zu}$ bestehen haben. In der Wiener Entomol. Zeitung, 1882, S. 65, zieht er Atomaria Degeerii Big. als Synonym zu Epidapus atomarius Deg.

H. L $0 \mathrm{ew}^{2}$ ) erhielt ein Weibchen Epidapus spec. aus Danzig, das aber mit der Beschreibung des venaticus Hal. nicht in allen Merkmalen übereinstimmte, weshalb Lo ew zögerte, es als diese Art $\mathrm{zu}$ bestimmen.

Van der $\mathrm{Wulp}^{3}$ ) erwähnt ein von Six im Rijzenburger Busch am 29. September 1868 erbeutetes Weibchen des Epidapus, das er trotz kleiner Abweichungen von der Beschreibung Schiners als venaticus Hal. anspricht.

Theobald endlich gibt 1892 (An account of British Flies) eine Beschreibung des Genus Epidapus, die mit Halidays Gattungsdiagnose übereinstimmt.

Schließlich sei noch erwähnt, daß Bezzi ${ }^{4}$ ) in einem Verzeichnisse von Fliegen mit fehlenden oder verkürzten Flügeln bei den Mycetophiliden (Fungivoriden) auch Epidapus atomarius Deg. (= Atomaria Degeerii Big.), als fragliche Art Epidapus venaticus Hal. und außerdem noch Epidapus scabies Hopkins 1895 anführt.

Die ge ographische Verbreitung der Gattung ist also eine sehr beschränkte, denn außer aus Irland (Hal.) und aus der Schweiz (Deg.), ist sie nur noch aus Deutschland (Loew und Winnertz) und aus Holland (Van der Wulp) bekannt. In anderen Sammelverzeichnissen wird sie nicht erwähnt. Trotzdem halte ich sie für eine weitverbreitete Gattung, die nur deshalb so selten genannt wird, weil das winzig kleine Tierchen leicht übersehen und nur durch Zucht erhalten werden kann.

1) Verh. d. zool. bot. Ges. Wien, 31. Bd. (1881), S. 355.

$\left.{ }^{2}\right)$ Über einige bei Danzig gefangene Dipteren, bei denen die Flügel verkümmert sind oder ganz fehlen. Schriften der Naturforsch. Ges. in Danzig. Neue Folge (1866), Heft 3 u. 4.

$\left.{ }^{3}\right)$ Tijdschr. voor Entomologie, 11. B. (1868), S. 224. - Diptera Neerlandica (1877), S. 107. Siehe auch: Wulp-de Meijere, Nieuwe Naamlijst voon Nederlandsche Diptera (1898).

${ }^{4}$ ) Sulla presenza del genere Chionea Dalm. in Italia e la reduzione dell'ali nei Ditteri. Reale Instit. Lombardo dei scienze e letteri. Rediconti. Ser. II, 33. Bd. (1900), S. 510 ff. 
Auch ich verdanke die Auffindung des Weibchens und die Entdeckung des bisher noch nicht beschriebenen Männchens nur einem glücklichen Zufalle.

Am 6. April 1915 entdeckte ich in der großen WypustekHöhle bei Brünn in Mähren, die ich mit Bewilligung der Gruppe für Höhlenforschung im Vereine deutscher Touristen in Brünn mehrmals besuchen konnte, ein Insekt, das ziemlich rasch über ein faules Holzstïck hinlief, das ich zur Untersuchung vom Boden aufgenommen hatte. Ich nahm eine größere Partie der ganz verfaulten Holzstïcke, die im finsteren Teile der Höhle, dem sogenannten Labyrinth, auf dem feuchten Boden umherlagen, in Papiersäcken mit nach Hause.

Bei der näheren Untersuchung erwies sich das gefangene Insekt als das Weibchen einer völlig flügel- und schwingerlosen Fliege, die ich nach den Diagnosen Schiners und W innertz' als Epidapus spec. erkannte.

Die mitgebrachten faulen Holzstïckchen wurden noch an demselben Tage in 3 Zuchtgläser gebracht, diese durch Glasplatten sorgfältig verschlossen und an einen dunklen Ort gestellt. $\mathrm{Zu}$ meiner Freude erhielt ich im Verlaufe der Monate April bis Juli zahlreiche Mücken, und zwar ungeflügelte Weibchen und zahlreiche geflügelte Männchen. Die letzteren waren stets zahlreicher als die Weibchen. Am 12. April, an welchem Tage ich die Zuchtgläser zum erstenmal untersuchte, erhielt ich aus dem 1. Glase: $12 \delta^{\nearrow} \sigma^{7}$ und $1 \%$, aus dem 2. Glase: $15 \sigma^{\top} \sigma^{\top}$ und 2 o $q$, aus dem 3. endlich $9 \sigma^{\pi} \sigma^{\pi}$, aber kein $\bigcirc$. Auch bei allen späteren Untersuchungen war die Zahl der ausgeschlüpften Männchen immer bedeutend größer als die der Weibchen. Am 6. Juni erhielt ich aus allen 3 Zuchtgläsern zusammen: 36 o o und 7 \% 9 .

Obwohl die Männchen völlig normale Flügel besitzen, machen sie von denselben (wie wohl die meisten Lycoriiden) äußerst selten Gebrauch; nur, wenn sie am Rande des Zuchtglases saßen und von hier verscheucht wurden, flogen sie auf. Ihr Flug ist langsam und schwerfällig; dafür laufen sie schnell und flink wie die flügellosen Weibchen.

Es unterliegt keinem Zweifel, daß die ausgeschlüpften geflügelten Mücken als Männchen zu den ungeflügelten Weibchen gehören, denn ich habe aus dem mitgebrachten Naterial, auf dem auch zahlreiche Milben und Collembolen lebten, nur diese eine L y c o ri i d e (Sciaride) erhalten. Die Zusammengehörigkeit der geschlüpften Männchen mit den Weibchen ergibt sich aber auch aus den zahlreichen gemeinsamen Merkmalen beider Geschlechter. 
Dadurch ist auch die Vermutung Schiners (1. c., S. 416, Fußnote), „daß Epidapus das flügellose Weibchen irgend einer SciaraArt sei", wenigstens teilweise bestätigt worden. Übrigens hatte die Behauptung Schiners an Wahrscheinlichkeit bedeutend gewonnen, seit Hopkins 1895 einen neuen Epidapus (scabies) aus Nordamerika beschrieben hat, dessen Weibchen ungeflügelt sind, während die Männchen normale Flügel besitzen.

Von der Gattung Epidapus Hal. sind bisher nur zwei paläarktische Formen beschrieben: atomarius Deg. und venaticus Hal. Eine dritte, von Hopkins veröffentlichte Art (E. scabies), stammt aus Nordamerika.

Es galt nun zunächst zu untersuchen, ob Epidapus venaticus Hal. nicht mit $E$. atomarius Deg. zusammenfalle, eine Vermutung, die schon Mik l. c. ausgesprochen hatte; auch Bezzi führt in seinem oben angeführten Verzeichnisse Epidapus venaticus Hal. als fragliche Art an.

Ich fand in den Beschreibungen der beiden Autoren zunächst verschiedene Angaben über die Färbung des Insekts. Hảliday nennt seinen venaticus „black“, Degeer aber spricht von seiner Tipula atomaria als einer „braungraulichten Erdmücke“. Beide Angaben können indessen richtig sein. Frisch ausgeschlüpfte Stücke sind nämlich immer ganz licht gefärbt und der Hinterleib des Weibchens zeigt kein Pigment, sondern ist zur Gänze von graulich-schmutzigweißer Farbe, während der Rückenschild bräunlich gefärbt ist. Später, und zwar sehr bald nach dem Ausschlüpfen, geht diese Farbe auf dem Rückenschilde in beiden Geschlechtern in eine schwarzbraune, etwas glänzende Färbung über, die Tergiten des Rückenschildes nehmen ebenfalls eine etwas glänzende, schwarze Farbe an, zwischen ihnen bleibt aber beim Weibchen noch die blasse, schmutzigweiße Membran sichtbar, so daß der Hinterleib ein geringeltes Aussehen erhält. Auch bei den vollkommen ausgefärbten Weibchen bleiben die Rüickenschilder des Hinterleibes durch diese Membran immer etwas getrennt. Auf diese "Zeichnung" des Hinterleibes macht schon van der W ulp aufmerksam. (Tijdschr. voor Entom., XI, 1868, Taf. 11, Fig. 1.)

Sieht man nun von den verschiedenen Angaben bezüglich der Färbung ab, so stimmt die Beschreibung der Tip. atomaria Dege ers sehr gut mit den Beschreibungen des venaticus bei Haliday und Winnertz überein. Nur in einer wesentlichen Angabe unterscheidet sich seine Beschreibung von der der beiden anderen Autoren. Er sagt: „An dem ganz runden Kopfe sitzen . . zwei sehr lange, überall gleich dicke, wenigstens $15 \mathrm{gliedrige} \mathrm{Fühlhörner".}$ 
Ep. venaticus Hal. hat aber $2+14$ gliedrige Fühler. Nun gewinnt aber die Ansicht Miks 1881: „Es ist nicht unmöglich, daß diese Angabe auf einem Irrtum beruht", sehr an Wahrscheinlichkeit, wenn man beachtet, daß D egeer von wenigstens 15gliedrigen Fühlern spricht. Da ihm nur ein einziges Weibchen vorgelegen ist, kann dem von ihm gebrauchten Worte nur die Bedeutung zukommen, daß die Fühler aus nicht weniger, wohl aber aus mehr als 15 Gliedern zusammengesetzt sein könnten, d. h. D e geer hat, da die Fühler bei dem untersuchten Exemplar vielleicht stark eingetrocknet waren und er die Zahl der Glieder nicht genau bestimmen konnte, selbst die Möglichkeit eines Irrtums zugeben wollen. Daß ein solcher Irrtum nicht ausgeschlossen ist, ersieht man auch daraus, daß die Fühler von Tipula atomaria in der Abbildung Degeers auf Tafel XLIV, Fig. 27 und 28, nur $2+12$ Glieder zeigen, so daß also auch zwischen der Beschreibung Degeers und der Abbildung ein Widerspruch besteht. Es ist daher als gewiß anzunehmen, daß atomarius Deg. und venaticus Hal. eine und dieselbe Art sind. Der jüngere Name Halidays muf dann als Synonym zu atomarius Deg. gezogen werden. Zweifelhaft blieb noch die Art, die Löw von Menge aus Danzig erhalten hatte und von der er mit Bezug auf Halidays kurze Beschreibung des venaticus 1837: „Black, slender; legs testaceous. $3 / 4$ "' " sagt:

„Meine Exemplare sind nicht schwarz, sondern braunrot, nur auf dem hintersten Drittel jedes Hinterleibsabschnittes dunkelbraun, die Beine sind nicht „testaceous", sondern samt den Hüften von blaßgelblicher Färbung; dieselbe blaßgelbliche Färbung haben die Fühler sowie die Taster und der Rüssel. Die Körperlänge beträgt eine volle Linie." Ferner hat seine Art deutliche Schienenspornen, während die Abbildung des Epidapus Hal. bei W a lker keine Spornen zeigt. Loew vermutet, daß das ihm zugesandte Weibchen vielleicht ein unausgefärbtes Stück sei.

Auch diese Frage konnte aber gelöst werden, und zwar auf Grund eines interessanten Fundes, den der bekannte Höhlenforscher Dr. Absolon im Mährischen Karste gemacht hat. Er fand im Jahre 1900 an der östlichen Lehne des Slouper Tales in einer Höhlenspalte, die mit Steinen, Moos und terra rossa ausgefüllt war, in Gemeinschaft mit zahlreichen Collembolen-Arten und anderen dämmerungsliebenden Tieren zwei kleine Arthropoden, die von ihm bei einer späteren Untersuchung als flügellose Dipteren erkannt und mir zur Bestimmung übergeben wurden. Die beiden Tiere (Weibchen) gehören der Gattung Epidapus an, aber sie weichen von atomarius (=vena-

Wien. Entom. Zeitung, XXXIV. Jhg., Heft VIII-X, Festschr. f. Reitter (22. Okt.1915). 
ticus) so sehr ab, daß sie als neue Art betrachtet werden müssen. Ich bin überzeugt, daß die von Dr. A b s o l o n entdeckte Art jene Spezies ist, welche H. L o e w vor sich hatte und von ihm nicht gedeutet werden konnte, da ihm nur die ungenügende Beschreibung Halidays vorlag.

Ich gebe nun nachstehend eine Beschreibung der Arten.

Gattung: Epidapus Hal. 1851.

Syn.: Atomaria Big. 1854.

? Corynoptera Winn. 1867 p. p.

Gattung sdiagnose: Corpus minimum. Antennae 16 - articulatae, in $\sigma^{7}$ corpore paulo longiores, articulis 2 basalibus subrotundis,

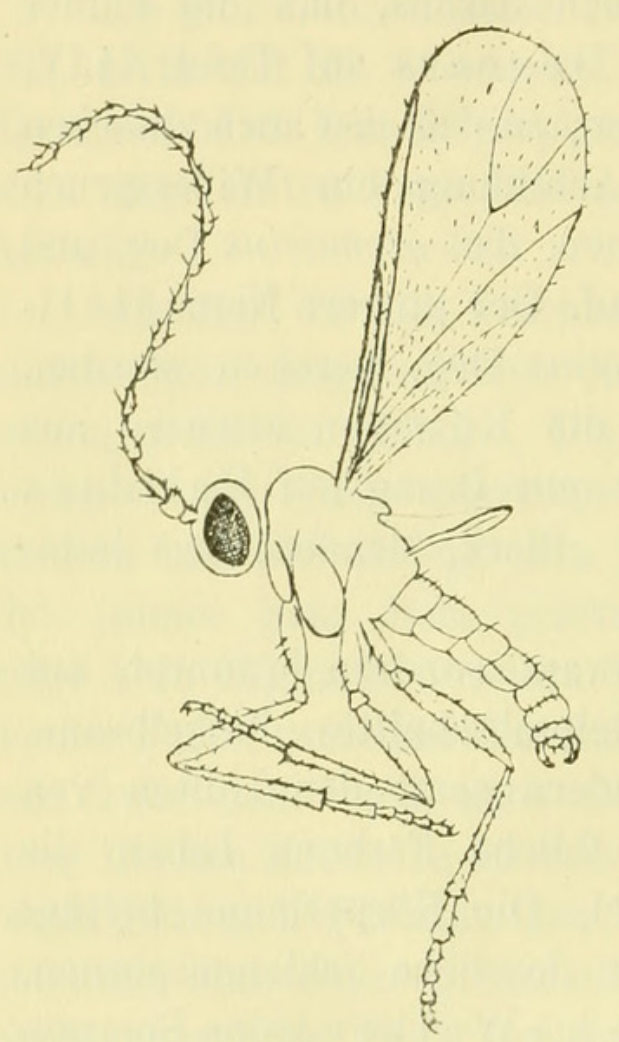

Fig. 1.

Epidapus atomarius $0^{2}$.

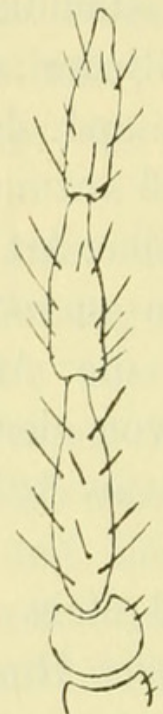

Fig. 2. Epid. atomarius ơ, Basalglied und die 3 ersten GeiBelglieder der

Fühler. articulis flagelli cylindricis, longe petiolatis, in utroque sexu verticillatis; antennae in $\mathrm{O}$ longitudine vix dimidii corporis, articulis flagelli breviter petiolatis et parum verticillatis.

Alae in o magnae, cuneatae, nervis costalibus distinctioribus, reliquis tenuibus.
Alae et halteres in o nulla.

Abdomen 7 -annulatum, pilosum vel nudum; in \& apud medium incrassaìum, apice decurvum; forceps in $\sigma^{7}$ parva, ut in Sciara instructa, oviductus in $q$ valvulis duabus obtusis pubescentibus instructum.

1. E. atomarius Deg. 1778.

Syn.: E. venaticus Hal. 1837.

? Corynoptera pumila Winn. 1867. O`.

Antennae in $\sigma^{7}$ corpore paulo longiores, flavae, $2+14$-articulatae, articulis basalibus subrotundis, articulis flagelli (1-13) cylindricis, petiolatis, verticillatis. Alae in on cuneatae, nervis ut in Sciara, sed nervo axillari rudimentario. $1.2 \mathrm{~mm}$.

Männchen (Fig. 1): Sehr kleine, schwarzbraune, in frischen Stücken mehr oder minder gelblich oder gelbbraun gefärbte Art. 
K opf rund, schwarz, Netzaugen groß.

Stirne mit einigen kurzen, sehr kleinen, nach vorne gerichteten Härchen besetzt. Rüssel und Taster kurz, gelb, letztere 4gliedrig.

F ü hler (Fig. 2) länger als der Körper, gelblich, $2+14$ gliedrig; das 1. Basalglied napfförmig, das 2. fast kugelförmig, beide Glieder mit wenigen stärkeren Börstchen besetzt. Die Geißelglieder gelblich oder gelbbraun (bei trockenen Stücken), zylindrisch; das erste Geißelglied fast um die Hälfte länger als das folgende, mit längeren Haarbörstchen dicht besetzt, die aber nicht so regelmäßig angeordnet sind, wie dies bei den folgenden Gliedern der Fall ist. Die übrigen Geibelglieder (2-13) ziemlich lang gestielt, mit zahlreichen, kranzförmig oder wirtelförmig gestellten Härchen dicht besetzt. Das letzte Fühlerglied ziemlich lang, ungestielt, länglich oval und ebenfalls mit Börstchen reich besetzt.

Rückenschild oben schwarzbraun, fast unbehaart, etwas glänzend, an den Seiten gelblich- oder gelbbraun. Schildchen klein, schwarz, Hinterrïcken gelbbraun.

Hüften ziemlich lang, an der Spitze mit einigen auffallenden Borsten besetzt, sowie die Sc hen kel, Schien en und Tars en von blaßgelber Farbe. Die ganzen Beine sind mikroskopisch fein, aber ziemlich dicht und lang behaart, die Schienen tragen vor der Spitze kleine, von der Behaarung durch größere Länge und Stärke ausgezeichnete, aber wenig auffallende Endspornen. Der Metatarsus der Füße ist doppelt so lang als das folgende Tarsenglied; das Endglied trägt zwei kleine Klauen; Haftballen mikroskopisch klein.

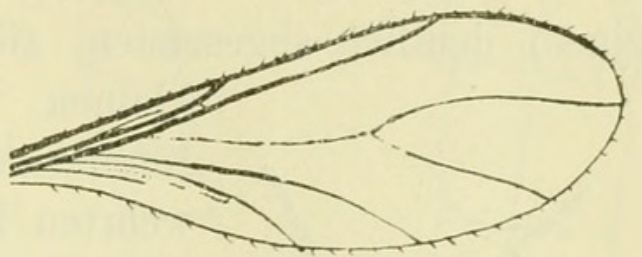

Fig. 3.

Epid. atomarius ơ, Flügel.

Flügel (Fig. 3) glasartig, am ganzen Rande kurz gewimpert, auf der Flügelfläche mikroskopisch fein behaart, im Umrisse keilförmig, ohne Flügellappen. Im Ruhezustande liegen sie dem Leibe parallel auf und überragen den Körper an Länge.

Die Randader verläuft in gleicher Stärke fast bis zur Flügelspitze. Die erste Längsader (Subcostalis) mündet weit vor der Gabelspitze der Discoidalis in die Randader. Die Mediastinalis ist kurz und endet in der Flügelfläche. Die dritte Längsader ${ }^{1}$ ) (Cubitalis) mündet weit vor der Spitze des Flügels in die Randader. Subcostalis und Cubitalis sind stärker als die anderen Adern (im Sciaridenflügel die Regel) und mit zerstreuten, nicht regelmäßig stehenden, stärkeren Börstchen schütter besetzt. Die Querader, welche die 1. und 3. Längsader verbindet, sehr undeutlich und schwer wahrnehmbar.

1) Die zweite Längsader (Radialis) fehlt im Sinne Schiners.

Wien. Entom. Zeitung, XXXIV. Jhg., Heft VHI-X, Festschr. f. Reitter (22. Okt. 1915). 
Vierte Längsader (Discoidalis) sehr schwach; Gabelstiel so undeutlich, daß er zu fehlen scheint; ebenso fehlt oft der Anfang der Gabelzinken, so daß nur zwei Drittel des oberen und unteren Gabelastes auf der Flügelfläche deutlich hervortreten. Fünfte und sechste

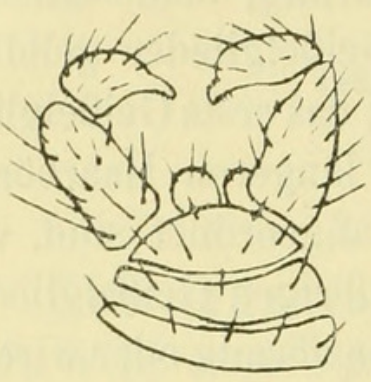

Fig. 4. Längsader (Posticalis und Analis) verlaufen in flachem Bogen zum Flügelhinterrand; letztere ist von einer Flügelfalte begleitet. Siebente Längsader (Axillaris) rudimentär.

Schwinger groß, keulenförmig, mit langem Stiel, von blasser, schmutzigweißer Farbe.

Hinterleib 7ringlig; vom Rücken geEpid. atomarius $\sigma^{\lambda}$, Zange. sehen nehmen die Endsegmente an Breite allmählich ab. ${ }^{1}$ )

Die Farbe des Hinterleibes ist im allgemeinen schwärzlich, mit einigem Glanze. Das erste Drittel der vorderen Segmente ist gelblichbraun, die Seiten und die Unterseite des Hinterleibes sind immer lichter gefärbt. Die Zange (Fig. 4) ist klein, gelblich. Die Endlamellen und die Zangenarme sind mit langen Börstchen und feinen Haaren dicht, stellenweise fast zottig behaart; jeder Zangenarm endet in einem deutlich abgesetzten, ziemlich spitzen, an der Innenseite mit kleinen, kur-

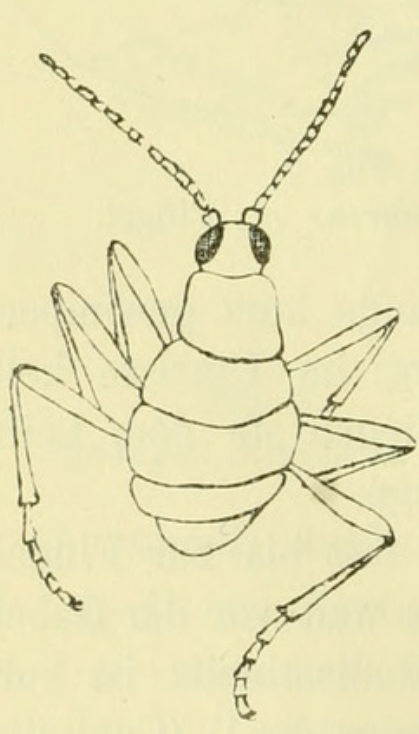

Fig. 5.

Epidapus atomarius 우, Rückenansicht, schematisch. zenStacheln bewehrten Zahn. Unter den Endlamellen treten zwei pustelförmige, blaßgelbe, mit einigen Haaren besetzte Anhänge hervor.

Größe: $1 \cdot 2 \mathrm{~mm}$.

W e ibch en (Fig. 5 und 6): Schwarzbraun, unreife Stücke gelblichgrau oder braun gelb.

K o p f rund, Netzaugen groß. Auf der Stirne einige, wenig auffallende, nach vorne gerichtete, sehr kurze Härchen. Rüssel und Taster kurz, gelb, letztere 4gliedrig.

1) In der schematischen Zeichnung (Fig. 1) ist das Ende des Hinterleibes mit der Zange nach oben gedreht, so daß das Hinterleibsende so breit erscheint wie die ersten Ringe, was der Wirklichkeit nicht entspricht. 
F ühler kaum von halber Körperlänge (wenn die Legeröhre mit den letzten Hinterleibsringen ganz ausgestreckt ist, dann messen die Fühler kaum ein Drittel der Länge des ganzen Körpers), $2+14$ gliedrig, die Basalglieder wie beim Männchen gebaut, die Geißelglieder kürzer als beim $\sigma^{\sigma}$, nicht so auffallend gestielt, mit Haaren kranzförmig besetzt.

Rückenschild oben schwarzbraun, glänzend, vor dem Schildchen eingedrückt, kurz und schütter behaart. Brustseiten von lichterer Färbung, kahl.

Hüften und Beine gelblichblaß oder gelblichbraun, sonst wie beim Männchen gebaut.

Flügel und Schwinger fehlen.

Hinterleib 7ringelig, oben schwärzlich, etwas glänzend, an den Seiten und auf der Unterseite gelblich, ziemlich stark und auffallend behaart. Bei den meisten Stïcken bleibt zwischen den schwarzen Tergiten am Hinterrande der Segmente die blasse, schmutzigweiße Membran sichtbar. Von oben gesehen erscheint der Hinterleib in der Mitte aufgetrieben, die folgenden Segmente nehmen an Breite rasch ab. Die letzten zwei Abschnitte des Hinterleibes sind samt der Legeröhre eingebogen, so daß das Tier von oben gesehen kurz und gedrungen erscheint; die Legeröhre (Fig. 6) wird im Leben immer gegen den Hinterleib zurückgeschlagen getragen. Sie endet mit zwei Paar kurzen, an der Spitze abgerundeten Lamellen von blasser, schmutzigweißer Farbe.

Größe: $1-1.2 \mathrm{~mm}$.

Larve (Fig. 7): $2 \mathrm{~mm}$ lang, 12ringelig, stielrund; Kopf glänzend

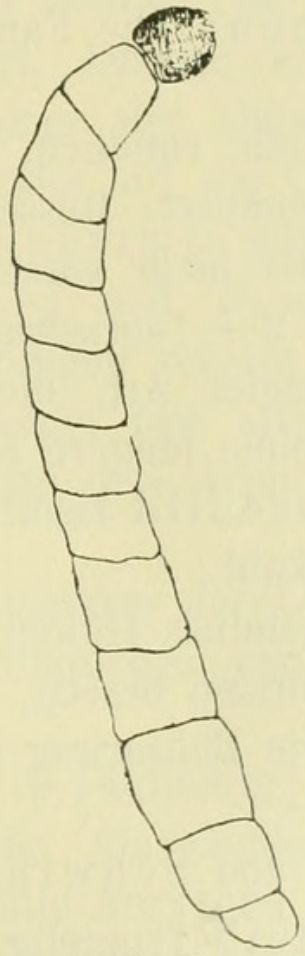

Fig. 7. Epidapus atomarius, Larve.

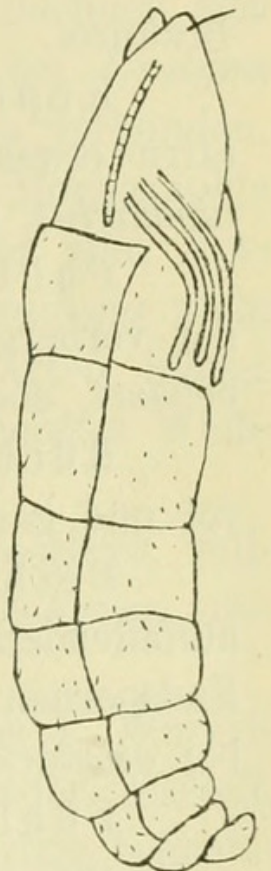

Fig. 8. Epidapus atomarius, Nymphe ㅇ. braunschwarz, der Körper glänzend weiß mit durchscheinendem Darmkanal.

Die Larve lebt in stark verfaultem Holze und kommt zur Verpuppung an die Oberfläche der Holzstïcke, wo sie sich in feinen Rissen zur Nymphe verwandelt. Die Puppenruhe dauert 7-9 Tage.

Nymphe (Fig. 8): ᄋ $1.3 \mathrm{~mm}$ lang, anfangs weißlich, später auf dem Kopfe und dem Thorax schwärzlichbraun. Stirne mit zwei

Wien. Entom. Zeitung, XXXIV. Jhg., Heft VIII-X, Festschr. f. Reitter (22. Okt. 1915). 
kleinen, schwarzen Börstchen; Fühler- und Fußscheiden gelblich, durchscheinend, so daß die Fühlerglieder und die Tarsen deutlich durchschimmern. Die Fufscheiden reichen bis zum zweiten Hinterleibssegment.

Hinterleib 7ringelig, blaßgelb, gegen hinten verschmälert, Endglied klein, etwas nach unten gekrümmt; die Ringe mit zahlreichen, auf Wärzchen aufstehenden Härchen dicht besetzt.

Eine ausführliche Beschreibung der Larve und Nymphe wird später folgen.

\section{E. Absoloni n. sp.}

Q : Similis praecedenti; colore fuscorubro, abdomine non piloso, fere nudo, antennis pedibusque breviter hirsutis, calcaribus tibiarum distinctioribus et terebra magis robusta ab eo differt. $1 \cdot 6-1.8 \mathrm{~mm}$.

Weibchen: Die Farbe des ganzen Körpers ist ein lichtes Braunrot.

K opf klein, rundlich, braunrot. Augen nierenförmig, auf der Stirn etwas genähert. Rüssel und Taster gelb und größer als bei atomarius, daher mehr vorstehend und deutlicher sichtbar.

F ïhler $2+14$ gliedrig, blaßgelblich, die Basalglieder wie bei der vorhergehenden Art, die Geibelglieder kurz gestielt, sehr fein behaart, aber ohne längere Haarbörstchen.

Rückenschild ziemlich kurz, oben und an den Seiten braunrot und ganz kahl.

B e in e gelblich, Hüften ziemlich lang, an der Spitze mit einigen auffallenden Borsten besetzt, Schienen an der Spitze mit deutlichen Endspornen. Die Behaarung der Beine ist viel zarter und feiner als bei atomarius.

Fühler und Schwinger fehlen.

Hinterleib 7ringelig, braunrot, in der Mitte aufgetrieben, gegen hinten verschmälert, fast ganz kahl.

Legeröhre robuster als bei atomarius, die Endlamellen dicker, aber im allgemeinen wie bei der vorhergehenden Art gebaut.

Größe: $1.6-1.8 \mathrm{~mm}$.

Das Männchen ist unbekannt. Dr. K. Absolon, dem zu Ehren ich die Spezies benannte, entdeckte die neue Art in einer kleinen Höhlenspalte des Slouper Tales im Mährischen Karst am 12. Mai 1900.

Epidapus Absoloni n. sp. unterscheidet sich von atomarius Deg. (=venaticus Hal.) durch die viel lichtere Farbe, die immer ins Braun- 
rote geht, eine Färbung, die bei atomarius nie vorkommt, durch die weniger lang behaarten, durch Börstchen nicht kranzförmig verzierten Fühler, durch die zarter behaarten Beine, die fast ganz fehlende Behaarung des Hinterleibes, durch die größeren und deutlicheren Endspornen der Schienen, durch die robustere Legeröhre und schließlich durch erheblichere Größe.

Es blieb nur noch die Frage offen, ob das von mir vorhin beschriebene Männchen des atomarius Deg. nicht von Winnertz in einer anderen Gattung beschrieben worden sei. Die Arten der Gattung Lycoria (Sciara) waren ausgeschlossen, da alle Sciara-Arten einen deutlichen Flügellappen besitzen. Aus demselben Grunde und infolge ihres Flügelgeäders kamen auch die Gattungen Trichosia, Cratyna und Zygoneura nicht in Betracht; bei ersterer ist die Flügelfläche deutlich (nicht mikroskopisch) behaart, letztere kennzeichnet sich durch die wellenförmig gebogenen Zinken der Gabel, bei Cratyna aber ist die Cubitalis durch eine Querader mit der Costa verbunden. Es blieben also nur noch die von Winnertz in seiner Monographie 1867 aufgestellten Genera: Corynoptera und Bradysia zum Vergleiche übrig. Von diesen beiden Gattungen scheint Bradysia nicht in Betracht zu kommen, da die Flügel fast aller Arten kürzer als der Hinterleib und die beschriebenen drei Arten überdies Weibchen sind.

Dagegen stimmt die Gattungsdiagnose der Corynoptera vollständig auf das oben beschriebene Männchen von Epidapus atomarius Deg.

„Fühler wie bei Sciara, also $2+14$ gliedrig; das 1 . Wurzelglied napfförmig, das 2. becherförmig, die Geißelglieder gestielt, walzenförmig, wirtelhaarig; Rückenschild kurz und dürftig behaart; Schienen ohne Seitendörnchen; Pulvillen äußerst klein, nur bei starker Vergrößerung wahrzunehmen, Flügel ohne Lappen, keulenförmig; die Gabel nicht gestreckt, weit offen." (Winnertz l. c.)

Nach diesen Angaben der Gattungsdiagnose, die alle auf atomarius $\sigma^{7}$ paften, war die Annahme nicht von der Hand zu weisen, daß irgend eine der 4 von Winnertz beschriebenen CorynopteraArten als Männchen zu Epidapus atomarius Deg. gehören könnte. Eine der Arten (minutula Winn.) ist ein Weibchen, bei perpusilla und gracilis, die nur im männlichen Geschlechte bekannt sind, wird ausdrücklich hervorgehoben, daß die Achselader (Axillaris) vollständig fehle. Die einzige Corynoptera-Art, welche zum Vergleiche heran-

Wien. Entom. Zeitung, XXXIV. Jhg., Heft VIII-X, Festschr. f. Reitter (22. Okt. 1915). 24 
gezogen werden kann, ist daher pumila, von der W innertz ebenfalls nur das Männchen beschreibt und die eine rudimentäre Achselader besitzt.

Die Beschreibung, die W innertz von dieser Art gibt, stimmt, abgesehen von kleinen Färbungsverschiedenheiten, fast in allen Punkten auf das Männchen des atomarius Deg. Nur die Angabe: „Fühler $1 \frac{1}{2}$ mal so lang als der Körper", macht es vorläufig unmöglich, beide Formen zu identifizieren. Was die Sache noch auffälliger macht, ist die Bemerkung Winnertz', daß er die vier Männchen seiner Corynoptera pumila aus Larven gezogen hat, die in einem faulen Stamme von Carpinus betulus lebten, dieselbe Bemerkung, die er bei Epidapus venaticus \& wörtlich wiederholt. Soll man annehmen, daß beide Formen (Corynoptera $\sigma^{\top}$ und Epidapus $\&$ ) aus e in er Zucht stammten, ohne daß Winnertz die Zusammengehörigkeit der Geschlechter erkannte? Diese Frage, ob Corynoptera Winn. teilweise zu Epidapus zu ziehen sei, kann endgültig wohl erst dann entschieden werden, wenn man Gelegenheit hat, die Type von Corynoptera pumila einzusehen.

Epidapus atomarius Deg. ist neu für die Fauna ÖsterreichUngarns. Wie eingangs erwähnt, habe ich ihn in einer mährischen Höhle entdeckt und durch seine Entdeckung wird auch das Verzeichnis der aus mährischen Höhlen bisher durch Dr. Absolon ${ }^{1}$ ) bekannten Dipteren um eine sehr interessante, weil flügellose Art, vermehrt, außer Chionea, die einzige ungeflügelte Fliege, die bisher in Höhlen überhaupt angetroffen wurde. Ihre Entdeckung in einer Höhle beweist natürlich noch nicht, daß sie als ein echtes Höhlentier zu betrachten und ihre Flügellosigkeit als Anpassung an das subterrane Leben anzusehen sei. Sie gehört aber zu jenen Fliegen, welche ihre ganze Verwandlung in der Höhle durchmachen und, einmal in die Höhle gelangt, nie mehr ans Tageslicht zurückkehren. Ihr häufiges Vorkommen in der Wypustek-Höhle scheint zu beweisen, daß sie die Vorliebe für Höhlen mit anderen Lycoriiden (Sciariden) teilt, die ich in den finstersten Räumen der mährischen Höhlen häufig angetroffen habe. Bei einer Lycoria-Art konnte ich auch feststellen, daf sie ihre ganze Verwandlung in der Höhle selbst durchmacht. Auf das häufige Vorkommen von Lycoriiden in Höhlen hat bereits $\mathrm{Bezzi}^{2}$ ) hingewiesen und er glaubt,

1) Bezzi M.: Ulteriore Notizie sulla Ditterofauna delle caverne. Atti della Società Italiana di Scienze Naturali XLVI, 1907.

${ }^{2}$ ) Diptères suive d'un Appendice sur les Diptères cavernicoles recueillis par le Dr. Absolon dans les Balcans. Archives de Zoologie expérimentale et generale. XLVIII, 1911, 5. Serie, VIII, Nr. 1. 
bei einer Art (Lycoria Absoloni) ein Zeichen der Anpassung an das subterrane Leben in der Kleinheit und Isolierung der chitinösen Tergiten und Sterniten und der Ausbreitung der weißen Hinterleibsmembran zwischen diesen Schildern gefunden zu haben, ein Nerkmal, das auch bei Phora aptina, Limosina Racovitzai und Gymnomus troglodytes anzutreffen ist. $\mathrm{Zu}$ dieser Auffassung kann ich mich heute noch nicht bekennen.

Für jeden Fall ist es aber interessant, daß die in Höhlen so häufigen Lycoriiden nun auch einen Vertreter der im weiblichen Geschlechte ungeflügelten Gattung Epidapus als Höhlenbewohner stellen können.

\section{Über Skulpturveränderungen auf Käferflügeldecken hervorgerufen durch Kochen.}

Von Franz Heikertinger, Wien.

Das Aufkochen von Käfern zwecks Ablösung vom Aufklebeblättchen, zur Vornahme anatomischer Untersuchungen, speziell zur Anfertigung von Penispräparaten, ist ein allgemein gebräuchlicher Vorgang. Als schweigend angenommene Voraussetzung gilt hiebei, daß Skulptur und Färbung des Käfers durch das Kochen keinerlei wesentliche Veränderungen erleiden.

Gelegentlich des Aufkochens von Haltica-Arten nun konnte ich die mich überraschende Beobachtung machen, daß es dennoch Fälle gibt, in denen das einfache, etwa 10 Sekunden währende Aufkochen eines Käfers in destilliertem Wasser einen wesentlichen, verändernden Einfluß auf die Skulptur der Elytren auszuüben imstande ist.

Ich beobachtete diese Erscheinung zuerst bei einer asiatischen Haltica-Art, die ich als deserticola Weise anspreche. Ich hatte aus etlichen kleinen Reihen von verschiedenen Fundorten $\sigma^{\top} \sigma^{\top}$ ausgewählt und Penispräparate angefertigt. Gelegentlich eines Vergleiches der Skulptur innerhalb einer Reihe fiel mir nun auf, daf das aufgekochte $\sigma^{\top}$ sich sehr stark von den übrigen Stücken ( $\sigma^{\top} \sigma^{\top}$ und $q$ ㅇ unterschied. In der Meinung, der Zufall habe mir ein einzelnes fremdes, nicht in die Serie gehörendes Stück in die Hand gespielt wählte ich ein anderes $\delta^{\nearrow}$, überzeugte mich, daß es hinsichtlich seiner Deckenskulptur nicht von den übrigen Stücken der Reihe abwich, kochte es auf und - vor mir lag wieder jenes fremdartig skulptierte Tier.

Die eingetretene Veränderung der Skulptur läßt sich kurz ungefähr folgendermaßen charakterisieren:

Wien. Entom. Zeitung, XXXIV. Jhg., Heft VIII - X, Festschr. f. Reitter (22. Okt. 1915) $21^{*}$ 


\section{$2 \mathrm{BHL}$ Biodiversity Heritage Library}

Czižek, Karl. 1915. "Über die im weiblichen Geschlechte ungeflügelte und schwingerlose Dipterengattung Epidapus HAL." Wiener entomologische Zeitung 34, 365-377. https://doi.org/10.5962/bhl.part.10630.

View This Item Online: https://www.biodiversitylibrary.org/item/43833

DOI: https://doi.org/10.5962/bhl.part.10630

Permalink: https://www.biodiversitylibrary.org/partpdf/10630

\section{Holding Institution}

Smithsonian Libraries

\section{Sponsored by}

Smithsonian

\section{Copyright \& Reuse}

Copyright Status: NOT_IN_COPYRIGHT

This document was created from content at the Biodiversity Heritage Library, the world's largest open access digital library for biodiversity literature and archives. Visit BHL at https://www.biodiversitylibrary.org. 